\title{
Nonexistence of Certain Spherical Designs of Odd Strengths and Cardinalities*
}

\author{
P. Boyvalenkov, ${ }^{1}$ D. Danev, ${ }^{1}$ and S. Nikova ${ }^{2}$ \\ ${ }^{1}$ Institute of Mathematics and Informatics, Bulgarian Academy of Sciences, \\ 8 G. Bonchev Street, 1113 Sofia, Bulgaria \\ peter@moi.math.bas.bg \\ 2 Department of Mathematics and Informatics, \\ V. Turnovo University, 5000 V. Turnovo, Bulgaria
}

\begin{abstract}
A spherical $\tau$-design on $\mathbf{S}^{n-1}$ is a finite set such that, for all polynomials $f$ of degree at most $\tau$, the average of $f$ over the set is equal to the average of $f$ over the sphere $\mathbf{S}^{n-1}$. In this paper we obtain some necessary conditions for the existence of designs of odd strengths and cardinalities. This gives nonexistence results in many cases. Asymptotically, we derive a bound which is better than the corresponding estimation ensured by the DelsarteGoethals-Seidel bound. We consider in detail the strengths $\tau=3$ and $\tau=5$ and obtain further nonexistence results in these cases. When the nonexistence argument does not work, we obtain bounds on the minimum distance of such designs.
\end{abstract}

\section{Introduction}

Spherical designs were introduced in 1977 by Delsarte et al. [9]. A nonempty finite subset $C$ of the Euclidean sphere $\mathbf{S}^{n-1}$ is called a spherical $\tau$-design if and only if the equality

$$
\int_{\mathbf{S}^{n-1}} f(x) d \mu(x)=\frac{1}{|C|} \sum_{x \in C} f(x)
$$

holds for any polynomial $f(x)=f\left(x_{1}, x_{2}, \ldots, x_{n}\right)$ of total degree at most $\tau$. Here $\mu(\cdot)$ is the normalized Lebesgue measure, i.e., $\mu\left(\mathbf{S}^{n-1}\right)=1$.

\footnotetext{
* This research was partially supported by the Bulgarian NSF under Contract MM-502/95. D. Danev is currently with the Department of Electrical Engineering, Linköping University, Linköping S-58183, Sweden. danyo@isy.lin.se.
} 
The Gegenbauer (ultraspherical) polynomials [1, Chapter 22] can be defined by

$$
\begin{gathered}
P_{0}^{(n)}(t)=1, \quad P_{1}^{(n)}(t)=t, \\
(i+n-2) P_{i+1}^{(n)}(t)=(2 i+n-2) t P_{i}^{(n)}(t)-i P_{i-1}^{(n)}(t) \quad \text { for } \quad i \geq 1 .
\end{gathered}
$$

If $f(t)=\sum_{i=0}^{k} a_{i} t^{i}$ is a real polynomial, then $f(t)$ can be uniquely expanded in terms of these polynomials as $f(t)=\sum_{i=0}^{k} f_{i} P_{i}^{(n)}(t)$. It is known that

$$
\begin{aligned}
f_{0} & =\left(\int_{-1}^{1}\left(1-t^{2}\right)^{(n-3) / 2} d t\right)^{-1} \int_{-1}^{1} f(t)\left(1-t^{2}\right)^{(n-3) / 2} d t \\
& =a_{0}+\sum_{i=1}^{[k / 2]} \frac{a_{2 i}(2 i-1) ! !}{n(n+2) \cdots(n+2 i-2)} .
\end{aligned}
$$

A second characterization of spherical $\tau$-designs (see, for example, Equation 1.10 of [10]) is that for any $\tau$-design $C \subset \mathbf{S}^{n-1}$ and for any point $y \in C$ the equality

$$
\sum_{x \in C \backslash\{y\}} f((x, y))=|C| f_{0}-f(1)
$$

holds for every real polynomial $f(t)$ of degree at most $\tau$, where $(x, y)$ is the usual inner product in $\mathbf{R}^{n}$.

Denote by $B(n, \tau)$ (resp. by $\left.B_{\text {odd }}(n, \tau)\right)$ the minimum possible cardinality (resp. odd cardinality) of a $\tau$-design on $\mathbf{S}^{n-1}$. The following Fisher-type lower bound on $B(n, \tau)$ was obtained by Delsarte et al. [9, Theorems 5.11 and 5.12]:

$$
\begin{aligned}
& B(n, \tau) \geq R(n, \tau) \\
& = \begin{cases}\left(\begin{array}{c}
n+e-1 \\
n-1
\end{array}\right)+\left(\begin{array}{c}
n+e-2 \\
n-1
\end{array}\right) & \text { if } \tau=2 e, \\
2\left(\begin{array}{c}
n+e-1 \\
n-1
\end{array}\right) & \text { if } \tau=2 e+1 .\end{cases}
\end{aligned}
$$

A spherical design is called tight if it attains the bound (4). Bannai and Damerell [3], [4] proved that for $n \geq 3$ tight spherical $\tau$-designs on $\mathbf{S}^{n-1}$ do not exist if $\tau=2 e$ and $e \geq 3$ or $\tau=2 e+1$ and $e \geq 4$ except for $\tau=11, n=24$. There exist tight $\tau$-designs for $\tau=1,2,3$ in all dimensions. Exactly eight tight $\tau$-designs with $\tau \geq 4$ are known. The bound (4) is therefore improved by one in the cases where the nonexistence of tight designs is proved (see [3]-[5]). Further improvements (by more than one) of the bound (4) for some $\tau \geq 6$ were obtained in some dimensions in [6] and [7] by using linear programming. Fazekas and Levenshtein [10, p. 287] write that the problem of asymptotic improvements of (4) is one of the most important problems in the theory of polynomial metric spaces (one of which is the Euclidean sphere).

We first obtain restrictions on the distributions of the inner products of $\tau$-designs with odd strength $\tau$. To do this we use suitable polynomials in (3). This gives necessary conditions for the existence of designs of odd strength in terms of the strength $\tau$, the 
dimension $n$, and the cardinality $R(n, \tau)+k$. These conditions imply nonexistence results for designs with odd cardinalities (i.e., for odd $k$ ) in many cases.

For $\tau=3$, we prove the nonexistence of spherical 3-designs on $\mathbf{S}^{n-1}$ with $R(n, 3)+$ $k=2 n+k$ points for all odd $k<\left(2^{1 / 3}-1\right) n+p$, where $p=2\left(14-5.2^{1 / 3}-4.2^{2 / 3}\right) / 9 \approx$ 0.30018. Therefore, we have

$$
B_{\text {odd }}(n, 3) \geq\left(1+2^{1 / 3}\right) n+p \approx\left(1+2^{1 / 3}\right) n+0.30018
$$

while (4) gives $B_{\text {odd }}(n, 3) \geq 2 n+1$. On the other hand, Bajnok [2] has constructed 3designs on $\mathbf{S}^{n-1}$ with all odd cardinalities greater than or equal to $R(n, 3)+n / 2=5 n / 2$ for $n \geq 6$, to 11 for $n=3,4$, and to 15 for $n=5$.

For $\tau=5$, we prove the nonexistence of spherical 5-designs on $\mathbf{S}^{n-1}$ with $R(n, 5)+$ $k=n^{2}+n+k$ points for all odd $k<n^{2}\left(2^{1 / 5}-1\right) / 2+p_{1} n+p_{2}$, where $p_{1}=$ $\left(-5+7.2^{1 / 5}-2.2^{3 / 5}\right) / 10 \approx 0.00095$ and $p_{2} \approx 0.0428$. Thus

$$
B_{\text {odd }}(n, 5) \geq \frac{1+2^{1 / 5}}{2} n^{2}+\left(1+p_{1}\right) n+p_{2} \approx \frac{1+2^{1 / 5}}{2} n^{2}+1.00095 n+0.0428
$$

instead of $B_{\text {odd }}(n, 5) \geq n^{2}+n+1$ from (4). Then we describe a method for proving further nonexistence results and give some examples. For constructions of spherical 5-designs we refer to [11] and [13].

In general, Theorem 2.8 shows that for $\tau=2 e+1$ and for every positive $p<$ $\left(2^{1 / \tau}-1\right) / e$ ! there exists a constant $n_{0}=n_{0}(p)$ such that for $n \geq n_{0}$ there do not exist $\tau$-designs on $\mathbf{S}^{n-1}$ with cardinality $R(n, \tau)+k$ for all odd positive $k \leq p n^{e}$. Therefore,

$$
B_{\text {odd }}(n, 2 e+1) \geq \frac{1+2^{1 / \tau}}{e !} n^{e} \quad \text { as } \quad n \rightarrow \infty
$$

whereas (4) gives $B_{\text {odd }}(n, 2 e+1) \geq 2 n^{e} / e !$ as $n \rightarrow \infty$.

In three dimensions we rule out the first open cases by showing the nonexistence of 3designs with seven points and 5-designs with thirteen points. Bajnok [2] has constructed 3-designs on $\mathbf{S}^{2}$ with $m$ points for $m=8$ and all $m \geq 10$. Hardin and Sloane [11] and Reznick [13] have constructed 5-designs on $\mathbf{S}^{2}$ with $m$ points for $m=12,16,18,20$, and all $m \geq 22$ and conjectured that the remaining cardinalities are impossible.

When our nonexistence argument does not work, we obtain bounds on the maximal inner product $s(C)=\max \{(x, y): x, y \in C, x \neq y\}$ of $(2 e+1)$-designs on $\mathbf{S}^{n-1}$ of odd sizes in terms of $e, n$, and $|C|$. These bounds are equivalent to bounds on the minimum distance $d(C)=\min \{d(x, y): x, y \in C, x \neq y\}$.

Fazekas and Levenshtein [10, Theorem 4] note that a combination of Levenshtein's bound for spherical codes and (4) implies a lower bound on the maximal possible inner product of spherical $\tau$-designs. For $\tau=2 e+1$, the asymptotic form of this bound is

$$
s(C) \geq \sqrt{\frac{2}{n}} h_{e}+O\left(n^{-3 / 2}\right) \quad \text { as } \quad n \rightarrow \infty,
$$

where $h_{e}$ is the greatest zero of the Hermite polynomial $H_{e}(t)$. For odd $\tau=2 e+1$ and $k=\gamma n^{e}$, we show that

$$
s(C) \geq \frac{1-2 \gamma e !-\gamma^{2}(e !)^{2}}{(1+\gamma e !)^{2}} \quad \text { as } \quad n \rightarrow \infty,
$$


which is positive for $\left(2^{1 / \tau}-1\right) / e !<\gamma<(\sqrt{2}-1) / e$ ! , and therefore is better for large enough $n$ than the bounds which can be obtained by the Fazekas-Levenshtein observation.

\section{General Necessary Conditions and Nonexistence Results}

For a $\tau$-design $C \subset \mathbf{S}^{n-1}$ and $y \in C$ we denote $I(y)=\{(x, y): x \in C, x \neq y\}$ counting with the multiplicities. Thus we may assume that $I(y)=\left\{t_{1}, t_{2}, \ldots, t_{|C|-1}\right\}$ where

$$
-1 \leq t_{1} \leq t_{2} \leq \cdots \leq t_{|C|-1}<1
$$

Then (3) becomes

$$
\sum_{i=1}^{|C|-1} f\left(t_{i}\right)=|C| f_{0}-f(1)
$$

and we use it in this form.

Delsarte et al. [9] obtain bound (4) by using suitable polynomials in the following so-called linear programming bound (see also Chapter 9 of [8]) for spherical designs.

Theorem 2.1 [9]. Let $n \geq 2$, let $\tau \geq 1$, and let $f(t)$ be a real polynomial such that

(A1) $f(t) \geq 0$ for $-1 \leq t \leq 1$, and

(A2) the coefficients in the Gegenbauer expansion $f(t)=\sum_{i=0}^{k} f_{i} P_{i}^{(n)}(t)$ satisfy $f_{\tau+1} \leq$ $0, \ldots, f_{k} \leq 0$.

Then $B(n, \tau) \geq f(1) / f_{0}$.

Let $\tau=2 e+1$ be odd, let $C \subset \mathbf{S}^{n-1}$ be a $\tau$-design of cardinality $|C|=R(n, \tau)+k$, and let $y \in C$. We first derive an upper bound on the least inner product $t_{1} \in I(y)$. We set

$$
g(t)=\left[P_{e}^{(n+2)}(t)\right]^{2}
$$

and

$$
\delta=-\frac{R(n, \tau)}{R(n, \tau)+2 k}<0 .
$$

Theorem 2.2. We have $t_{1} \leq \delta$.

Proof. The bound (4) for $\tau=2 e+1$ was obtained [9, Theorem 5.12] by using the polynomial $(t+1) g(t)$ in Theorem 2.1. Since $g(t)$ is an even function, (1) shows that the first coefficients in the Gegenbauer expansions of $(t+1) g(t)$ and $g(t)$ coincide. We denote this common coefficient by $g_{0}$ (in fact, $2 g(1) / g_{0}=2 / g_{0}=R(n, \tau)$ by Theorem 5.12 of [9]). 
We set $f(t)=\left(t-t_{1}\right) g(t)=(t+1) g(t)-\left(t_{1}+1\right) g(t)$ in (3). Then the left-hand side is nonnegative, and the right-hand side is

$$
f_{0}|C|-f(1)=-t_{1} g_{0}(R(n, \tau)+k)-1+t_{1},
$$

which implies $t_{1} \leq-R(n, \tau) /(R(n, \tau)+2 k)=\delta$.

We denote by $b_{i}$ the first coefficient in the Gegenbauer expansion of $t^{i}$ (see (1) and (2)).

Lemma 2.3. We have $b_{i}=(i-1) ! ! / n(n+2) \cdots(n+i-2)$ for $i$ even and $b_{i}=0$ for $i$ odd.

For $\varepsilon \in[-1,0)$, we say that the point $x$ is $\varepsilon$-near antipodal of $y$, if $(x, y) \leq \varepsilon$. For $\varepsilon=-1$, this is the usual antipodality.

Theorem 2.4. Let $n \geq 3$, let $\tau=2 e+1 \geq 3$, and let $k \geq 1$ be such that

$$
b_{2 e}[R(n, \tau)+k]-1<2 \delta^{2 e} .
$$

Then each point of $C$ has a unique $\delta$-near antipode from $C$. In particular, $k$ must be even.

Proof. If $t_{2} \leq \delta$ for some $y \in C$, then by $f(t)=t^{2 e}$ in (3) we obtain

$$
\begin{aligned}
b_{2 e}|C|-1 & =\sum_{i=1}^{|C|-1} t_{i}^{2 e} \\
& \geq 2 t_{2}^{2 e} \\
& \geq 2 \delta^{2 e}
\end{aligned}
$$

which contradicts (5). Therefore

$$
t_{1} \leq \delta<t_{2}
$$

for all $y \in C$. Then for any point $y \in C$ there exists a unique $\delta$-near antipode $x \in C$ (in our notations, $x=x_{1}$ ). Therefore the points of $C$ must be divided into disjoint pairs, i.e., $|C|$ is even. Since $R(n, \tau)$ is even for $\tau$ odd, the number $k$ must be even.

Corollary 2.5. If $n \geq 3$ and the odd numbers $\tau=2 e+1 \geq 3$ and $k \geq 1$ are such that (5) is satisfied, then there exist no spherical $\tau$-designs on $\mathbf{S}^{n-1}$ with $R(n, \tau)+k$ points.

Setting different polynomials in (3) and using the estimation from Theorem 2.2 one can obtain better nonexistence results for $(2 e+1)$-designs with odd cardinalities. In the next two sections the cases $e=1$ and $e=2$ are considered in detail. Before that we give another universal nonexistence rule. 
Theorem 2.6. Let $\alpha$ be the least zero of the polynomial $g(t)$. If $n \geq 3$ and the odd numbers $\tau=2 e+1 \geq 3$ and $k \geq 1$ satisfy the conditions

$$
\delta<\alpha
$$

and

$$
-2 \delta g(\delta)>1
$$

then there do not exist $\tau$-designs on $\mathbf{S}^{n-1}$ with $R(n, \tau)+k$ points.

Proof. We suppose that $t_{2} \leq \delta$ for some $y \in C$. The even function $g(t)$ decreases in the interval $[-1, \alpha], g(1)=1$, and $g_{0}=2 / R(n, \tau)$. Thus

$$
\begin{aligned}
-\frac{1}{\delta} & =\frac{R(n, \tau)+2 k}{R(n, \tau)} \\
& =g_{0}|C|-g(1) \\
& =\sum_{i=1}^{|C|-1} g\left(t_{i}\right) \\
& \geq 2 g\left(t_{2}\right) \\
& \geq 2 g(\delta),
\end{aligned}
$$

which contradicts (7). Then $t_{2}>\delta$ for all $y \in C$ and the nonexistence argument from Theorem 2.4 can be applied.

Conditions (5) and (7) coincide when $e=1$ as we see in Theorem 3.1 and Example 3.2. For $e \geq 2$, (7) gives stronger results, but we have to check if (6) holds. In fact, we conjecture that (7) implies (6). This is suggested by the cases $\tau=3$ (see Theorem 3.1 and Example 3.2) and $\tau=5$ (see Theorem 4.3).

Lemma 2.7. If $k$ and $\tau$ are fixed and $n \rightarrow \infty$, then $\alpha$ tends to zero.

Proof. By the recurrence relation for the Gegenbauer polynomials, one has

$$
P_{e}^{(n)}(t) \stackrel{n \rightarrow \infty}{\longrightarrow} t P_{e-1}^{(n)}(t)
$$

whence $P_{e}^{(n)}(t) \stackrel{n \rightarrow \infty}{\longrightarrow} t^{e}$ and we are done. Another proof follows by the explicit formula [1, p. 775] for the Gegenbauer polynomials (all but the leading coefficients tend to zero when $n$ tends to infinity).

We now discuss the nonexistence results ensured by Theorem 2.6 for $\tau$ fixed and $n \rightarrow \infty$.

Theorem 2.8. For fixed $\tau=2 e+1 \geq 3$ and every positive $p<p_{0}=\left(2^{1 / \tau}-1\right) / e$ ! there exists a constant $n_{0}=n_{0}(p)$ such that for $n \geq n_{0}$ there do not exist $\tau$-designs on $\mathbf{S}^{n-1}$ with cardinality $R(n, \tau)+k$ for all odd positive $k \leq p n^{e}$. 
Proof. Since $R(n, \tau)=2(n+e-1) \cdots(n+1) n / e ! \approx 2 n^{e} / e !$ as $n \rightarrow \infty$, we have

$$
\begin{gathered}
\delta \leq-\frac{R(n, \tau)}{R(n, \tau)+2 p n^{e}} \\
\stackrel{n \rightarrow \infty}{\longrightarrow}-\frac{1}{1+p e !}<0 .
\end{gathered}
$$

Thus (6) is satisfied for large enough $n$. For (7) we have

$$
\begin{aligned}
-2 \delta g(\delta) & \geq \frac{2 R(n, \tau)}{R(n, \tau)+2 p n^{e}} \cdot g\left(\frac{R(n, \tau)}{R(n, \tau)+2 p n^{e}}\right) \\
& \stackrel{n \rightarrow \infty}{\longrightarrow} \frac{2}{(1+p e !)^{\tau}} \\
& >\frac{2}{\left(1+p_{0} e !\right)^{\tau}} \\
& =1
\end{aligned}
$$

(use, as in the proof of Lemma 2.7, that $P_{e}^{(n)}(t) \stackrel{n \rightarrow \infty}{\longrightarrow} t^{e}$ ). This completes the proof.

Corollary 2.9. For fixed $\tau=2 e+1 \geq 3$ and $n \rightarrow \infty$, we have

$$
B_{\text {odd }}(n, \tau) \geq \frac{1+2^{1 / \tau}}{e !} n^{e} .
$$

The above approach can be further refined and improved. We show this in the next two sections (see the discussions after Example 3.2 and Corollary 4.5).

\section{Nonexistence of Certain 3-Designs}

For $n=3$, we have $R(n, 3)=2 n, g(t)=t^{2}, \delta=-n /(n+k)$, and $\alpha=0$. Let $C \subset \mathbf{S}^{n-1}$ be a 3-design with $|C|=R(n, 3)+k=2 n+k$ points and $y \in C$. We set $C=\left\{y, x_{1}, x_{2}, \ldots, x_{2 n+k-1}\right\}$ and $\left(x_{i}, y\right)=t_{i}$ for $i=1,2, \ldots, 2 n+k-1$, where $-1 \leq t_{1} \leq t_{2} \leq \cdots \leq t_{2 n+k-1}$. By Corollary 2.5 we obtain the following:

Theorem 3.1. For odd $k<\left(2^{1 / 3}-1\right) n \approx 0.26 n$ there exist no spherical 3-designs on $\mathbf{S}^{n-1}$ with $2 n+k$ points.

Proof. For $\tau=2 e+1=3$, (5) is equivalent to $k<\left(2^{1 / 3}-1\right) n$.

Example 3.2. There exist no 3 -designs with $2 n+1$ points $(k=1)$ in dimensions $n \geq 4$, 3 -designs with $2 n+3$ points $(k=3)$ in dimensions $n \geq 12$, etc. Notice that Theorem 2.6 gives the same result because $P_{1}^{(n+2)}(t)=t$ (and $\alpha=0>\delta=-n /(n+k)$ ).

To obtain further nonexistence results for spherical 3-designs we need better estimations. As a simple consequence, we prove the nonexistence of spherical 3-designs on $\mathbf{S}^{2}$ with seven points which resolves the first open case. 
Lemma 3.3. For every real a we have

$$
t_{1} \leq F(a)=\frac{h_{1}(a)}{h_{2}(a)}=-\frac{n a^{2}+2(n+k) a+n}{n(2 n+k-1) a^{2}+2 n a+n+k} \leq t_{2 n+k-1} .
$$

Proof. Set $f(t)=(t-a)^{2}\left(t-t_{i}\right)$, where $i=1$ or $2 n+k-1$, in (3). The left-hand side is nonnegative for $i=1$ and nonpositive for $i=2 n+k-1$. By (2), we compute

$$
f_{0}=a^{2} t_{i}-\frac{1}{n}\left(2 a+t_{i}\right) .
$$

Resolving the inequalities $f_{0}|C|-f(1) \geq 0$ for $i=1$ and $f_{0}|C|-f(1) \leq 0$ for $i=2 n+k-1$, we obtain the desired estimations.

The argument in Section 2 corresponds to $a=0$ in Lemma 3.3. Now we investigate the function $F(a)$. The equation $F^{\prime}(a)=0$ is equivalent to the quadratic equation

$$
n(n+k-1) a^{2}+n(n-1) a-k=0 .
$$

Let $a_{1}$ and $a_{2}$ be the negative and positive root of (8) respectively, i.e.,

$$
a_{1,2}=\frac{-n(n-1) \mp \sqrt{n^{2}(n-1)^{2}+4 n k(n+k-1)}}{2 n(n+k-1)} .
$$

Lemma 3.4. We have $t_{1} \leq F\left(a_{2}\right)=a_{1}<0$ and $t_{2 n+k-1} \geq F\left(a_{1}\right)=a_{2}>0$.

Proof. The function $F(a)$ has maximum for $a=a_{1}$ and minimum for $a=a_{2}$. Since $F^{\prime}\left(a_{1}\right)=0$, we have $F\left(a_{1}\right)=h_{1}^{\prime}\left(a_{1}\right) / h_{2}^{\prime}\left(a_{1}\right)$. To check the identity $F\left(a_{1}\right)=a_{2}$, apply the Viète formulas and a little algebra. Analogously, $F\left(a_{2}\right)=a_{1}$.

We now obtain a necessary condition for the existence of 3-designs which in fact refines (5).

Theorem 3.5. If $k$ is odd, then

$$
a_{1} \geq-\frac{2 n^{3}+(5 k-7) n^{2}+\left(4 k^{2}-15 k+5\right) n+k(k-1)(k-5)}{2 n\left[2 n^{2}+(k-5) n+3(1-k)\right]} .
$$

Proof. If $t_{2}>a_{1}$ for all $y \in C$ we can apply the nonexistence argument from Corollary 2.5. Therefore $t_{2} \leq a_{1}$ for some $y \in C$.

We set $f(t)=(t-a)^{2}$ in (3) assuming $a \geq a_{1}$. We have (compute $f_{0}$ by (2))

$$
\begin{aligned}
(2 n+k-1) a^{2}+2 a+\frac{n+k}{n} & =f_{0}|C|-f(1) \\
& =\sum_{i=1}^{2 n+k-1}\left(t_{i}-a\right)^{2} \\
& \geq 2\left(t_{2}-a\right)^{2} \\
& \geq 2\left(a_{1}-a\right)^{2} .
\end{aligned}
$$


This gives

$$
(2 n+k-3) a^{2}+2\left(1+2 a_{1}\right) a+\frac{n+k}{n}-2 a_{1}^{2} \geq 0 .
$$

The last quadratic function has its minimum at the point $a_{0}=-\left(1+2 a_{1}\right) /(2 n+k-3) \geq$ $a_{1}$. This minimum equals

$$
-\frac{2}{2 n+k-3}\left((2 n+k-1) a_{1}^{2}+2 a_{1}+\frac{1}{2}-\frac{(n+k)(2 n+k-3)}{2 n}\right)
$$

whence

$$
(2 n+k-1) a_{1}^{2}+2 a_{1}+\frac{1}{2} \leq \frac{(n+k)(2 n+k-3)}{2 n} .
$$

Since $a_{1}$ is a root of (8), we express $a_{1}^{2}$ from (8) to obtain a linear inequality with respect to $a_{1}$ which is equivalent to (9).

Corollary 3.6. There exist no spherical 3-designs with seven points on $\mathbf{S}^{2}$.

Proof. In this case $(9)$ is violated since $a_{1}=-(1+\sqrt{2}) / 3 \approx-0.804$ while the right-hand side of (9) is equal to $-\frac{1}{2}$.

Example 3.2 and Corollary 3.6 complete the case $k=1$, i.e., we have shown the nonexistence of spherical 3-designs on $\mathbf{S}^{n-1}$ with $2 n+1$ points (what is the first possible cardinality of a nontight 3-design?) in all dimensions $n \geq 3$. The precise investigation of condition (9) implies the following result which slightly improves Theorem 2.8 for $\tau=3$.

Corollary 3.7. There exist no spherical 3-designs on $\mathbf{S}^{n-1}$ with $R(n, 3)+k=2 n+k$ points for $n \geq 3$ and all odd positive $k<n\left(2^{1 / 3}-1\right)+p$, where $p=2\left(14-5.2^{1 / 3}-\right.$ $\left.4.2^{2 / 3}\right) / 9 \approx 0.30018$. In other words,

$$
B_{\text {odd }}(n, 3) \geq\left(1+2^{1 / 3}\right) n+p \approx\left(1+2^{1 / 3}\right) n+0.30018 .
$$

Proof. We are interested in the pairs $(n, k)$ for which $n \geq 3$ and $k \geq 1$. In what follows we take only such pairs under consideration. After a routine calculation (which we made using Maple V), inequality (9) takes the form

$$
\begin{aligned}
0 \leq h_{3}(n, k)= & k^{6}+4(2 n-3) k^{5}+2\left(13 n^{2}-39 n+23\right) k^{4} \\
& +2\left(21 n^{3}-93 n^{2}+112 n-30\right) k^{3} \\
& +\left(31 n^{4}-186 n^{3}+349 n^{2}-210 n+25\right) k^{2} \\
& +2 n\left(2 n^{4}-25 n^{3}+77 n^{2}-82 n+28\right) k \\
& -n^{2}\left(4 n^{4}+20 n^{3}-33 n^{2}+22 n-5\right) .
\end{aligned}
$$

The constant $p$ was "conjectured" by setting $k=\left(2^{1 / 3}-1\right) n+p$, forgetting the small (with respect to the degrees of $n$ ) terms and resolving a linear (with respect to $p$ ) equation. 
In this case we get a polynomial of the variable $n$ of degree five with leading coefficient equal to $6\left(1+2^{1 / 3}\right)^{2} p+4\left(4-5.2^{1 / 3}\right)$. Since this coefficient must be nonpositive, we see that the largest $p$ which can be used is exactly $2\left(14-5.2^{1 / 3}-4.2^{2 / 3}\right) / 9$.

We have

$$
h_{3}\left(n, n\left(2^{1 / 3}-1\right)+p\right)<-15 n^{4}+49 n^{3}-32 n^{2}+5 n+1<0,
$$

whenever $n \geq 3$. The standard investigation of $h_{3}(n, k)$ shows that it is an increasing function of the variable $k$ in the interval $[1,+\infty)$. Thus, for every positive integer $k$, for which $k<n\left(2^{1 / 3}-1\right)+p$, we have $h_{3}(n, k)<h_{3}\left(n, n\left(2^{1 / 3}-1\right)+p\right)<0$. In this case, condition (9) is violated, that completes the proof.

Example 3.8. As noted in the Introduction, Bajnok [2] has constructed 3-designs on $\mathbf{S}^{n-1}$ with all odd cardinalities greater than or equal to $R(n, 3)+n / 2=5 n / 2$ for $n \geq 6$, to 11 for $n=3,4$, and to 15 for $n=5$, and with all possible even cardinalities. Thus Corollary 3.7 shows that all possible cardinalities of 3-designs on $\mathbf{S}^{n-1}$ are already known for $n=4,6$ and only one unsettled case remains in dimensions $n=3,5$, and $7 \leq n \leq 14$.

\section{Nonexistence of Certain 5-Designs}

Let $C \subset \mathbf{S}^{n-1}$ be a 5-design with $|C|=R(n, 5)+k=n^{2}+n+k$ points and $y \in C$. We set $C=\left\{y, x_{1}, x_{2}, \ldots, x_{n^{2}+n+k-1}\right\}$ and $\left(x_{i}, y\right)=t_{i}$ for $i=1,2, \ldots, n^{2}+n+k-1$, where $-1 \leq t_{1} \leq t_{2} \leq \cdots \leq t_{n^{2}+n+k-1}$. Theorem 2.2 gives $t_{1}<\delta=-n(n+1) /\left(n^{2}+n+2 k\right)$. Inequality (5) is equivalent to

$$
G(n, k)=\frac{n^{2}+n+2 k}{n(n+1)} \cdot \sqrt[4]{\frac{2 n^{2}+n+3 k}{2 n(n+2)}}<1 .
$$

Theorem 4.1. If $n \geq 3$ and the odd integer $k \geq 1$ are such that $G(n, k)<1$, then there exist no spherical 5-designs on $\mathbf{S}^{n-1}$ with $n^{2}+n+k$ points.

Example 4.2. For $k=1$, the investigation of the function $G(n, 1)$ implies the nonexistence of spherical 5-designs with $n^{2}+n+1$ points in all dimensions $n \geq 7$. Similarly, for $k=3$, one obtains the nonexistence of spherical 5-designs with $n^{2}+n+3$ points in all dimensions $n \geq 20$. In three dimensions, Theorem 4.1 provides no information.

We now discuss the nonexistence results ensured by Theorem 2.6 for $\tau=5$.

Theorem 4.3. For $n \geq 3$ and all odd positive $k<n(n+1)(\sqrt{n+2}-1) / 2$, there exist no spherical 5-designs on $\mathbf{S}^{n-1}$ if

$$
(n+1)\left(n^{2}+n+2 k\right)^{5}<2 n\left[n^{2}(n+1)^{3}-4 k\left(n^{2}+n+k\right)\right]^{2} .
$$


Proof. Since $P_{2}^{(n+2)}(t)=\left((n+2) t^{2}-1\right) /(n+1)$ (i.e., $\left.\alpha=-1 / \sqrt{n+2}\right)$, condition (6) is equivalent to $k<n(n+1)(\sqrt{n+2}-1) / 2$. A little algebra shows that (7) is equivalent to (10).

Example 4.4. For $k=1$, (10) implies the nonexistence of 5-designs with $n^{2}+n+1$ points in all dimensions $n \geq 4$ (in fact, after Example 4.2, we need to check (10) for $n=4,5$, and 6 only). Analogously, for $k=3$, one obtains the nonexistence of spherical 5-designs with $n^{2}+n+3$ points in all dimensions $n \geq 7$.

Investigation of condition (10) gives the following result:

Corollary 4.5. For $n \geq 3$, there exist no spherical 5-designs on $\mathbf{S}^{n-1}$ with $n^{2}+n+k$ points for all odd positive $k<p_{0} n^{2}+p_{1} n$, where $p_{0}=\left(2^{1 / 5}-1\right) / 2 \approx 0.074349$, $p_{1}=\left(-5+7.2^{1 / 5}-2.2^{3 / 5}\right) / 10 \approx 0.00095$.

Proof. Using Maple V again, we found that inequality (10) is equivalent to

$$
\begin{aligned}
0<h_{5}(n, k)= & -32(n+1) k^{5}-16\left(5 n^{2}+10 n+3\right) n k^{4} \\
& -16 n^{2}(n+1)\left(5 n^{2}+10 n+1\right) k^{3}-8 n^{3}(n+1)^{2}\left(5 n^{2}+12 n+3\right) k^{2} \\
& -2 n^{4}(n+1)^{4}(5 n+13) k+n^{5}(n+1)^{6} .
\end{aligned}
$$

It is immediate that, for every fixed positive number $n$, the function $h_{5}(n, k)$ of the variable $k$ is decreasing in $(0,+\infty)$. The constant $p_{1}$ was found in the same way as in the case $\tau=3$ (see Corollary 3.7). We now substitute $k$ with $p_{0} n^{2}+p_{1} n$ in $h_{5}(n, k)$ and obtain

$$
\begin{aligned}
h_{5}(n, k) & >h_{5}\left(n, p_{0} n^{2}+p_{1} n\right) \\
& >n^{9}+4 n^{8}+6 n^{7}+3 n^{6}>0
\end{aligned}
$$

for $k \in\left[1, p_{0} n^{2}+p_{1} n\right)$ and for every positive $n$. Thus (10) is satisfied for every positive $k<p_{0} n^{2}+p_{1} n$.

To complete the proof we check the additional condition in Theorem 4.3 by seeing that $p_{0} n^{2}+p_{1} n<n(n+1)(\sqrt{n+2}-1) / 2$ holds for every $n \geq 3$.

We have to mention that neither of the constants $p_{0}$ nor $p_{1}$ can be made larger by our method. Indeed, if we try to increase some of them, a negative coefficient will appear in the front of the highest power of $n$ in $h_{5}\left(n, p_{0} n^{2}+p_{1} n\right)$. In this case $h_{5}\left(n, p_{0} n^{2}+p_{1} n\right)$ will be negative for large enough $n$.

Using the last corollary, we get

$$
B_{\text {odd }}(n, 5) \geq \frac{1+2^{1 / 5}}{2} n^{2}+\left(1+p_{1}\right) n \approx \frac{1+2^{1 / 5}}{2} n^{2}+1.00095 n
$$

instead of $B_{\text {odd }}(n, 5) \geq n^{2}+n+1$ by (4). 
The interested reader can further apply (preferable by using Maple or Mathematica) the argument from the proof of Corollary 4.5. Indeed, one can prove that

$$
B_{\text {odd }}(n, 5) \geq \frac{1+2^{1 / 5}}{2} n^{2}+\left(1+p_{1}\right) n+p_{2} \approx \frac{1+2^{1 / 5}}{2} n^{2}+1.00095 n+0.0428
$$

In this case the function $h_{5}\left(n, p_{0} n^{2}+p_{1} n+p_{2}\right)$ is a polynomial which vanishes at $n=3$ and is positive for all $n>3$.

We do not know the optimal polynomials for obtaining nonexistence results by our method. In what follows in this section, we propose a way for improving (11).

To refine our approach, we have to consider in (3) (as in Section 3) the polynomials $f_{1}(t)=\left(t^{2}+a t+b\right)^{2}\left(t-t_{1}\right)$ and $f_{2}(t)=\left(t^{2}+a t+b\right)^{2}\left(t-t_{n^{2}+n+k-1}\right)$ for the best choices of the parameters $a$ and $b$. The following lemma is an analog of Lemma 3.3 and must be proved in the same way.

Lemma 4.6. For every real $a$ and $b$ we have

$$
\begin{aligned}
t_{1} \leq F(a, b) & =-\frac{n(1+a+b)^{2}-2 a\left(n^{2}+n+k\right)(b+3 /(n+2))}{\left(n^{2}+n+k\right)\left(a^{2}+n b^{2}+2 b+3 /(n+2)\right)-n(1+a+b)^{2}} \\
& \leq t_{n^{2}+n+k-1} .
\end{aligned}
$$

We describe a simple algorithm for proving further nonexistence results for 5-designs. Given $n \geq 3$ and odd $k \geq 1$, we first obtain by Lemma 4.6 some bounds $t_{1} \leq b_{1}$ and $t_{n^{2}+n+k-1} \geq b_{2}$. Then we search for polynomials $f(t)=(t-c)^{2}(t-d)^{\overline{2}}$, where $b_{1} \leq c \leq d \leq b_{2}$. The nonexistence argument can be applied if

$$
f_{0}|C|-f(1)<2 f(c)+f(d) .
$$

Of course, it is enough to find just one pair $(c, d)$ for which the last inequality holds. This can be checked by a computer for a few seconds. The algorithm works strongly enough to rule out the first open case.

Theorem 4.7. There exist no spherical 5-designs with 13 points on $\mathbf{S}^{2}$.

Proof. We obtain the bounds $t_{1} \leq-0.898=b_{1}$ and $t_{12} \geq 0.489=b_{2}$ by using the pairs $(a, b)=(-0.148,-0.167)$ and $(1.24,0.307)$, respectively. Then the pair $(c, d)=\left(-0.341, b_{2}\right)$ works.

The above technique works in other cases where (10) does not give nonexistence results. The interested reader can check the nonexistence of 5-designs with $45=R(6,5)+3$ points on $\mathbf{S}^{5}$.

\section{Bounds on the Maximal Inner Products of Designs with Odd Strengths and Cardinalities}

When the nonexistence argument from Theorem 2.4 does not work, we can obtain a lower bound on the maximal inner product $s(C)$ (equivalently, an upper bound on the 
minimum distance $d(C)=\sqrt{2(1-s(C))})$ of all $(2 e+1)$-designs $C \subset \mathbf{S}^{n-1}$ with odd cardinality $R(n, 2 e+1)+k$.

Theorem 5.1. Let $\tau=2 e+1 \geq 3$, let $k \geq 3$ be odd, let $n \geq 3$, and let $C \subset \mathbf{S}^{n-1}$ be a $\tau$-design with $R(n, \tau)+k$ points. Then

$$
s(C) \geq 2 \delta^{2}-1 \quad \text { and } \quad d(C) \leq 2 \sqrt{1-\delta^{2}} .
$$

Proof. There exists a point $y \in C$ such that $t_{2} \leq \delta$. Let the acute angle $\varphi$ be such that $\cos \varphi=-\delta$. Then the angle between the vectors $x_{1}$ and $x_{2}$ does not exceed $2 \varphi$. Thus we have

$$
s(C) \geq\left(x_{1}, x_{2}\right) \geq \cos 2 \varphi=2 \cos ^{2} \varphi-1=2 \delta^{2}-1 .
$$

The bound for $d(C)$ is obtained by $d(C)=\sqrt{2(1-s(C))}$.

Bounds on the maximal inner product can be obtained by a combination (see [10]) of Levenshtein's bounds on spherical codes [12] and the Delsarte-Goethals-Seidel bound (4). For any spherical $(2 e+1)$-design $C \subset \mathbf{S}^{n-1}$, a reformulation of Theorem 1 of [10] says that

$$
s(C) \geq t_{e}^{(n+2)}\left(\operatorname{resp} . d(C) \leq \sqrt{2\left(1-t_{e}^{(n+2)}\right)}\right),
$$

where $t_{e}^{(n+2)}$ is the greatest zero of the Gegenbauer polynomial $P_{e}^{(n+2)}(t)$.

The bound (12) has the following asymptotic form [10, Theorem 4]:

$$
s(C) \geq \sqrt{\frac{2}{n}} h_{e}+O\left(n^{-3 / 2}\right) \quad \text { as } \quad n \rightarrow \infty,
$$

where $h_{e}$ is the greatest zero of the Hermite polynomial $H_{e}(t)$ [1, Chapter 22]. The Hermite polynomials can be defined by

$$
H_{0}(t)=1, \quad H_{1}(t)=2 t, \quad H_{i+1}(t)=2 t H_{i}(t)-2 i H_{i-1}(t), \quad i \geq 1,
$$

and one has $h_{1}=0, h_{2}=1 / \sqrt{2}, h_{3}=\sqrt{\frac{3}{2}}, h_{e}=\sqrt{2 e}+O\left(e^{-1 / 6}\right)$ as $e \rightarrow \infty$. For $\tau$ fixed, the right-hand side of (13) tends to zero as $n$ tends to $\infty$. We obtain a positive lower bound on $s(C)$ that does not depend (explicitly) on $n$.

Theorem 5.2. Let $\tau=2 e+1$, let $k=\gamma n^{e}$ be odd, and let $\left(2^{1 / \tau}-1\right) / e !<\gamma<(\sqrt{2}-$ 1)/e!. Then for any spherical $\tau$-design $C \subset \mathbf{S}^{n-1}$ with odd cardinality $|C|=R(n, \tau)+k$ and as $n \rightarrow \infty$, we have

$$
s(C) \geq h(\gamma)=\frac{1-2 \gamma e !-\gamma^{2}(e !)^{2}}{(1+\gamma e !)^{2}}=\frac{2}{(1+\gamma e !)^{2}}-1 .
$$

Proof. For large enough $n$ we have $R(n, \tau) \approx 2 n^{e} / e$ ! and Theorem 5.1 implies the assertion. 
The function $h(\gamma)$ is strictly decreasing for $\gamma>0$. Since $h((\sqrt{2}-1) / e$ !) $=0$, we have $h(\gamma)>0$ for all $\left(2^{1 / \tau}-1\right) / e !<\gamma<(\sqrt{2}-1) / e$ !. Therefore, Theorem 5.2 gives better results than (12) for all large enough $n$.

Finally, we show some improvements of the bound (12) by Theorem 5.1.

Example 5.3. For $\tau=k=3$ and $n=8,9,10$ the bound (12) gives $s(C) \geq 0$, while Theorem 5.1 implies $s(C) \geq \frac{7}{121}, \frac{1}{8}, \frac{31}{169}$, respectively. For $\tau=5, k=3$, and $n=7,8$, (10) gives $s(C) \geq \frac{1}{3}, 1 / \sqrt{10}$ while our Theorem 5.1 gives $s(C) \geq \frac{607}{961}, \frac{119}{169}$, respectively.

\section{Acknowledgments}

The authors thank Stefan Dodunekov, Ventzislav Nikov, and an anonymous referee for helpful suggestions and comments.

\section{References}

1. M. Abramowitz, I.A. Stegun, Handbook of Mathematical Functions, with Formulas, Graphs and Mathematical Tables, Dover, New York, 1965.

2. B. Bajnok, Constructions of spherical 3-designs, Graphs Combin. 14 (1998), 97-107.

3. E. Bannai, R.M. Damerell, Tight spherical designs I, J. Math. Soc. Japan 31 (1979), 199-207.

4. E. Bannai, R.M. Damerell, Tight spherical designs II, J. London Math. Soc. 21 (1980), 13-30.

5. P.G. Boyvalenkov, Computing distance distributions of spherical designs, Linear Algebra Appl. 226/228 (1995), 277-286.

6. P.G. Boyvalenkov, S. Nikova, New Lower Bounds for Some Spherical Designs, Lecture Notes in Computer Science, vol. 781, Springer-Verlag, Berlin, 1994, pp. 207-216.

7. P.G. Boyvalenkov, S. Nikova, Improvements of the lower bounds for some spherical designs, Math. Balkanica, to appear.

8. J.H. Conway, N.J.A. Sloane, Sphere Packings, Lattices and Groups, 2nd edn., Springer-Verlag, New York, 1993.

9. P. Delsarte, J.-M. Goethals, J.J. Seidel, Spherical codes and designs, Geom. Dedicata 6 (1977), 363-388.

10. G. Fazekas, V.I. Levenshtein, On upper bounds for code distance and covering radius of designs in polynomial metric spaces, J. Combin. Theory Ser. A 70 (1995), 267-288.

11. R.H. Hardin, N.J.A. Sloane, McLaren's improved snub cube and other new spherical designs in three dimensions, Discrete Comput. Geom. 15 (1996), 429-441.

12. V.I. Levenshtein, Bounds for packings in metric spaces and some of their applications, Problemy Kibernet. 40 (1983), 44-110 (in Russian).

13. B. Reznick, Some constructions of spherical 5-designs, Linear Algebra Appl. 226/228 (1995), $163-196$.

Received January 30, 1997, and in revised form November 29, 1997. 